Article

\title{
Tracking Control for Piezoelectric Actuators with Advanced Feed-forward Compensation Combined with PI Control.
}

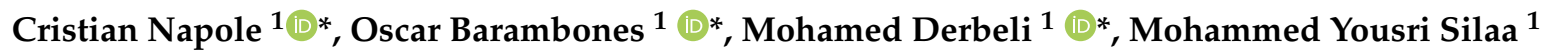 \\ (D), Isidro Calvo ${ }^{1}$ (D) and Javier Velasco ${ }^{2}$, \\ 1 System Engineering and Automation Deparment, Faculty of Engineering of Vitoria-Gasteiz, Basque Country \\ University (UPV / EHU); cristianmario.napole@ehu.eus (C.M.N.), oscar.barambones@ehu.eus (O.B.C.), \\ derbelimohamed1@gmail.com (M.D.), silaa.mohammed.yousri@gmail.com (M.Y.S.), isidro.calvo@ehu.eus \\ (I.C.) \\ 2 Fundación Centro de Tecnologías Aeronáuticas (CTA), Juan de la Cierva 1, 01510 Miñano, Spain; \\ javier.velasco@ctaero.com
}

\begin{abstract}
Piezoelectric actuators (PEA) are devices which can support large actuation forces compared to their small size and are widely used in high precision applications where micro- and nanopositioning is required. Nonetheless, these actuators have undeniable non-linearities were the well-known are creep, vibration dynamics and hysteresis. The latter mentioned is originated due to a combination of mechanical strain and electric field action; as a consequence, these can affect the PEA tracking performance and even reach instability. The scope of this paper is to reduce the hysteresis effect using and comparing different control strategies like feedback with feed-forward (FF) structure which is often used to compensate the non-linearities and diminish the errors due to uncertainties. In this research, black-box models were analysed; subsequently, a classic feedback control like proportional-integral (PI) was combined with the FF methods proposed separately and embedded into a dSpace platform to perform real-time experiments. Results were analysed in-depth in terms of the error, the control signal and the integral of the absolute error (IAE). It was found that with the proposed methods, the hysteresis effect could be diminished to acceptable ranges for high-precision tracking with a satisfactory control signal.
\end{abstract}

Keywords: Black-box modelling; Piezoelectric; Feedforward; Precise position; PI; PEA; hysteresis; feedback

\section{Introduction}

PEAs are high precision positioning systems with a wide range of usage such as active vibration systems for vehicles [1], sensing [2], energy recovery [3] and stick-slip motors [4]. These devices not only are accurate but also possess a broad stiffness in comparison to their size [5]. Despite the advantages, these actuators have hysteresis, which is a significant non-linearity effect which downgrades the performance. This feature, occurs as a combination between electric field, material and mechanical strain [6], that yields the PEAs to have a position-error up to $22 \%$ [7].

Along the years, the hysteresis can be reduced provided that proper control is well designed. Due to the simplicity and robustness, Sliding Mode Control (SMC) is a successful and widely used tool for PEAs [8]; for example, authors of [9] presented an SMC with a PID type sliding surface which was implemented in real-time. In their study, the authors used a PEA with an asymmetrical hysteresis and they designed the controller based on a state observer with a conventional Bouc-Wen (BW) model (which is inflexible for asymmetry [10]). Even though the accuracy was increased, SMC is naturally 
known for the chattering effect. Other model-based control strategies could have more accurate models like Prandtl-Ishlinskii or Preisach for state observation but these are widely complex to implement due to the inverse calculation. For instance, a Prandtl-Ishlinskii model was used in [11] which the calculation is purely mathematical and lacks of practicality.

Authors from [12] suggested that a feedback controller can handle errors in positioning as well as reduction of unknown effects; also, the addition of a FF amplifies the nonlinearity compensation. Black-box (BB) models are commonly used for FF compensation due to mapping ability for unknown dynamic systems [13], where the common structures are Artificial Neural Networks (ANNs), Hammerstein-Wiener (HW), etc. In [14] a sophisticated FF with feedback control was used: a linear approximation was done and it was compared with an ANN which was used for mapping; additionally, a conventional PI and a neural PID were embedded for the whole combination. Outcomes showed that a neural PID with an FF-ANN could improve accuracy. Even that ANNs could give satisfactory results for mapping, other BB strategies could be used. HW is an approach which uses both authors theories to identify non-linear dynamic models [15], where the structure consists of non-linear peripheral blocks and a central linear one. An application with an HW was implemented in [16], where the author used the HW block to model the non-linearities of a piezoelectric pad which was combined with a Kalman Filter to estimate the position that was controlled by a Fuzzy-PID. Results have shown an increment in the accuracy due to the complex structure.

In this research, we used a PI controller which parameters were optimised by reduction of the IAE; moreover, an ANN and an HW were designed in Matlab/ Simulink, contrasted and tested individually in a control architecture with a PI. In the Section 2, the hardware configuration is explained within the PEA specifications followed by a shallow description of the hysteresis and the control structures used during the experiments. Section 3 provides the results of the hysteresis fitting that the ANN and HW provided; also, the errors and actuation signal were interpreted for the control architectures. Finally, the Section 4 provides a summarize about the whole research and which future strategies could be analysed.

\section{Methods}

\subsection{Hardware description}

This study was based on commercial hardware from Thorlabs which was used for experiments. The PEA PK4FYC2 is a discrete stack actuator where the measurement is done by a full-bridge Wheatstone circuit. A technical description is provided in the Table 1.

Table 1. Thorlabs PK4FYC2 Technical Specifications

\begin{tabular}{ccc}
\hline Properties & Values & Units \\
\hline Physical Dimensions & $7.3 \times 7.3 \times 36$ & $\mathrm{~mm}$ \\
Nominal Max Displacement & 38.5 & $\mu \mathrm{m}$ \\
Max Force & 1000 & $\mathrm{~N}$ \\
Drive Voltage Range & $0-150$ & $\mathrm{~V}$ \\
Error due to hysteresis & 15 & $\%$ \\
\hline
\end{tabular}

The Figure 1 shows the hardware architectures in which the control strategies were embedded in a dSpace DSP1104 that provided a 0-10V signal which is sent to the PEA driver cube (KPZ101 by Thorlabs); this hardware amplifies the signal into 0-150V straight into the PEA. The measurement is done in two steps: the strain gauge measurement is augmented in a pre-amplifier AMP002 and linked into a strain gauge reader KPZ101 by Thorlabs. The latter provides a 0-10V measurement signal which is used in the DSP1104 hardware for control purpose. 


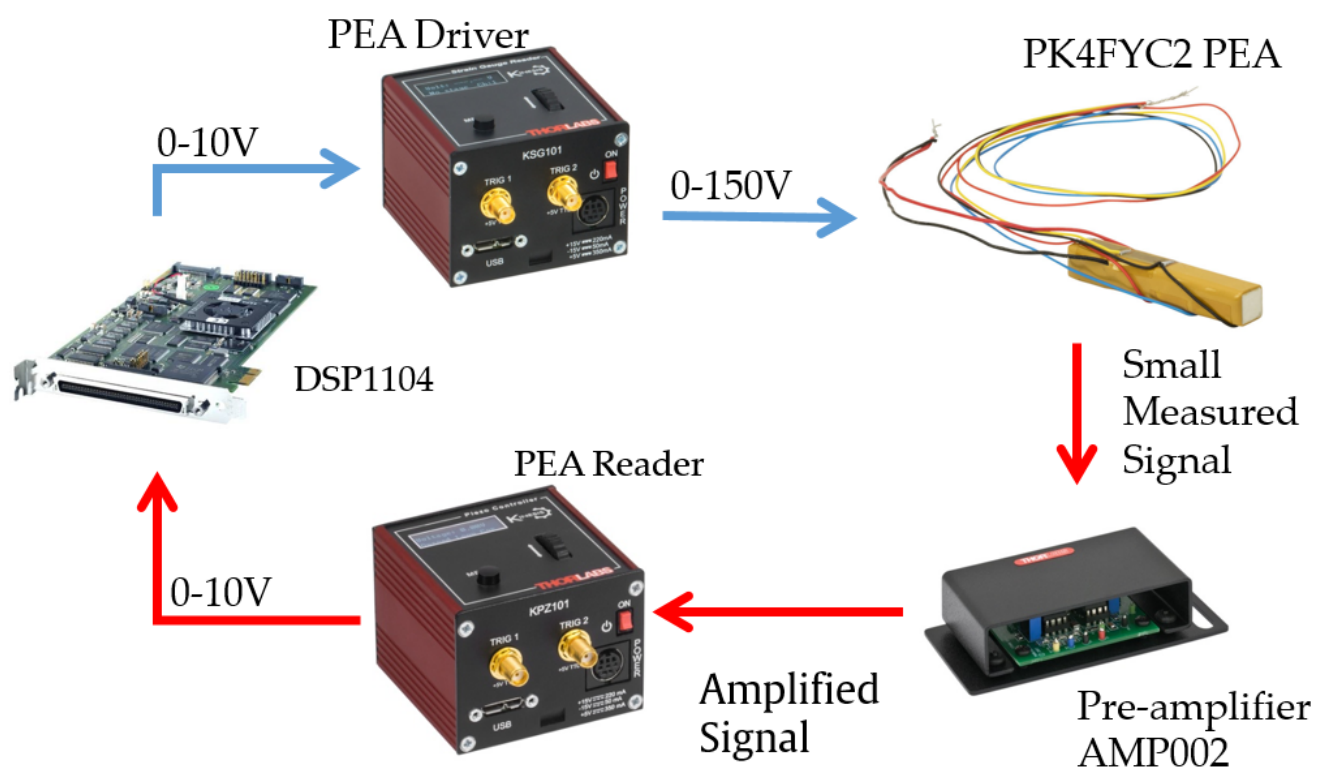

Figure 1. Hardware architecture

\subsection{PEA hysteresis}

The hysteresis was obtained through a triangle wave as input voltage; also, mathematically it represents the sum of infinite harmonics which implies that any proposed controller needs to grant high performance at tracking [17]. According to [14], the PEA used reflects a prominent hysteresis effect at the maximum driving voltage, so for the tests and to avoid damage, it was configured at 145 $\mathrm{V}$ and the period was set at 1 second. The sampling time was configured at $1 \mathrm{kHz}$ which reflects a suitable balance between acquisition and hardware limitation.

The Figure 2 is the PK4FY2 PEA hysteresis graph for two cycles at the start. Usually, the hysteresis tends to the converge the loop between (2) and (3) since the position only begins at zero whenever calibration is done before any operation.

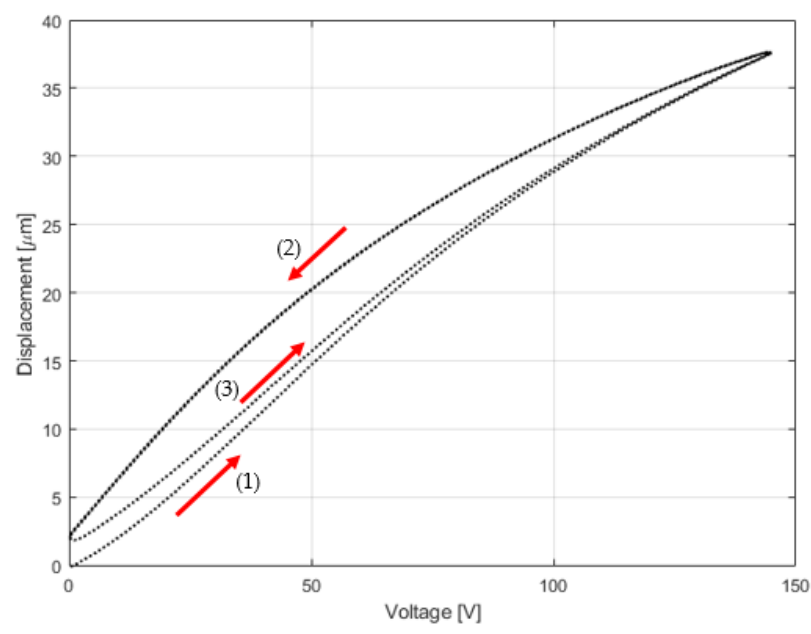

Figure 2. Piezoelectric hysteresis graph

\subsection{ANN configuration}

The first architecture used for mapping the PEA non-linearity was a Time Delay Neural Network (TDNN) which is a recurrent ANN [18] type. The structure implies that the predicted output depends on the past values of the input (which was set as 5 by default) and merged with a non-linear function $f$ 
as shown in Equation 1. The function $f$ was approximated by a non-linear combination of a hidden and output layer with its activation functions and weights. The Figure 3 displays the architecture of the TDNN where both layers have different weights and bias (in terms of $i$ and $j$ ). The configuration of the ANN was disposed as the one used by the author [14] where the number of neurons in the hidden layer was 22. The training information was set with 10 seconds of triangle waves which reflected the PEA displacement and voltage, that had to be as the input and output, respectively. Also, the data was divided into $70 \%$ for training, $15 \%$ in validation and the rest for testing. Furthermore, the training algorithm used was Levenberg-Marquardt (LM) as the Matlab ANN Toolbox suggests; the calculation was carried until acceptable values of mean squared error (MSE) were achieved.

$$
y(t)=f(u(t-1), \ldots, u(t-5))
$$

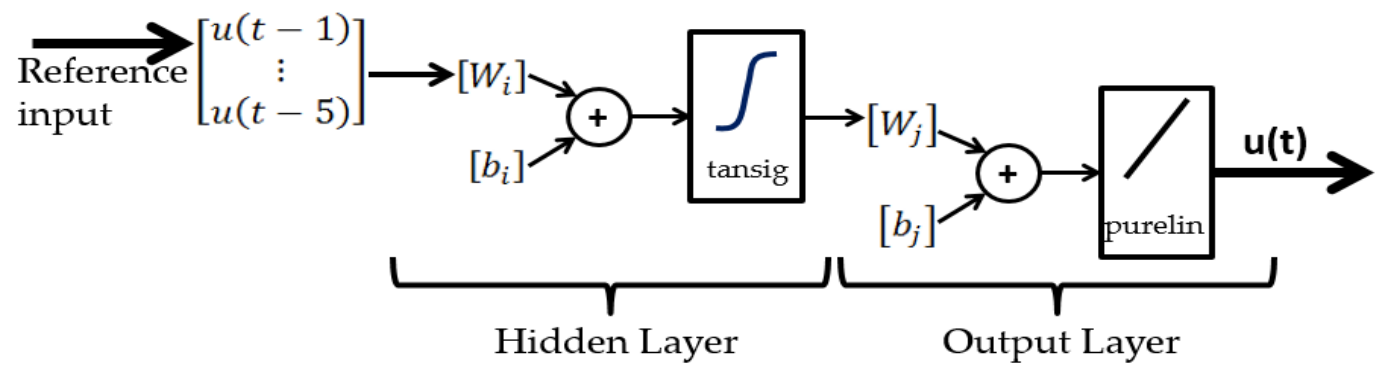

Figure 3. TDNN explicit architecture.

\subsection{Hammerstein-Wiener configuration}

As Figure 4 shows, HW consists of three blocks in series where the outer ones are used to map the input and output non-linearities whereas the middle block is a linear function (Equation 2) in terms of the time shift operator $q^{-1}$, such that $q^{-1} u(t)=u(t-T)$. The parameters $n_{b}, n_{f}$ and $n_{d}$ represents the degree of $\mathrm{B}, \mathrm{F}$ and the delay degree, respectively.

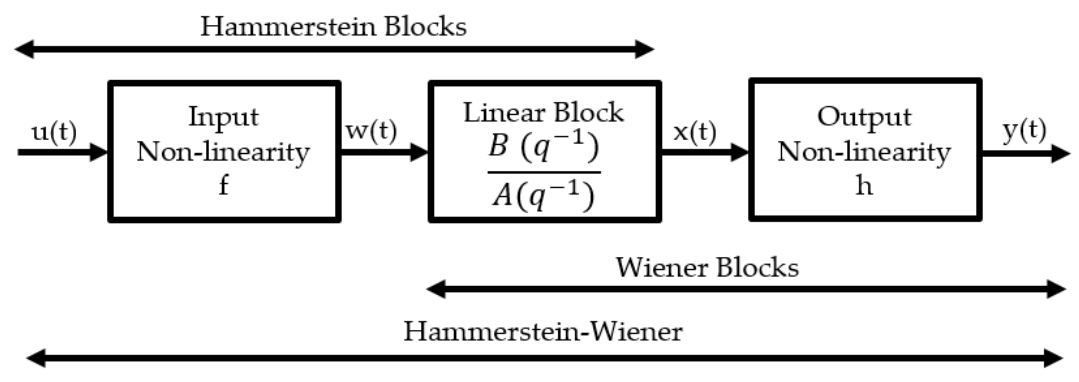

Figure 4. HW architecture

$$
\frac{B}{F}=q^{-n_{d}} \frac{\sum_{i=1}^{n_{b}} b_{i} q^{-i+1}}{1+\sum_{j=1}^{n_{f}} b_{j} q^{-j}}
$$

The parameters of the HW block were estimated with the MATLAB System Identification Toolbox which uses a loss function based on error reduction between the model output and measured response as a metric. The approximation algorithm was set to automatic choice according to the toolbox criterion. The non-linear blocks can be estimated with different functions (such as piece-wise functions, polynomial, wavelet, etc.) were the criterion used to determine which could suit best was the Fit Percent, that defines the quality of the model likeness to the measured data. 


\subsection{Control structure}

The experiments were done with two control structures that have the same PI but with an FF-ANN and an FF-HW (Figure 5). The PI tuning was based on the IAE (Equation 3 in real-time and aiming to reach the best performance. For a fair comparison, the constants $K_{p}$ and $K_{i}$ were the same in both architectures.

$$
I A E=\int_{0}^{\infty}|e(t)| d t
$$

(a)

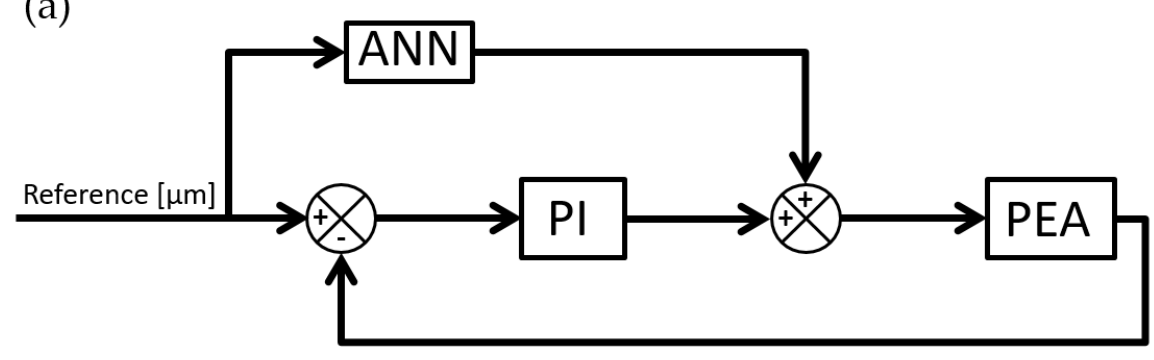

(b)

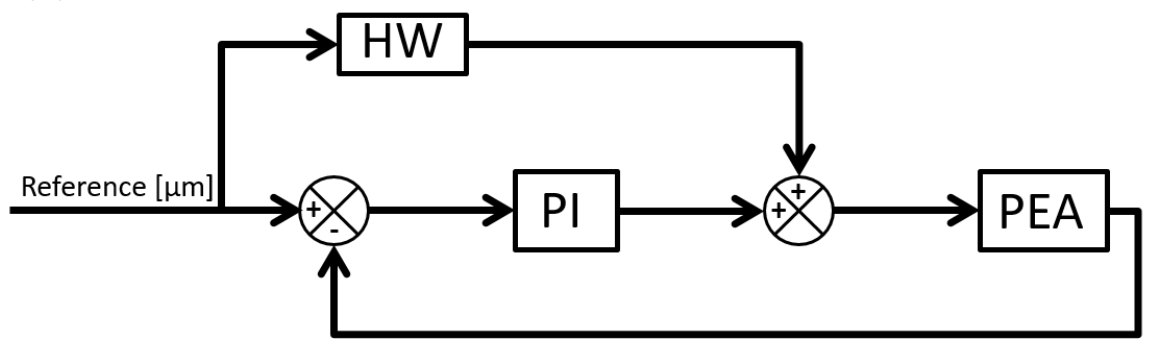

Figure 5. Control architectures where (a) uses a FF-ANN and (b) a FF-HW.

\section{Results and Discussion}

\subsection{Hysteresis fitting results}

Before testing the control structures, the accuracy of each FF block was tested and contrasted. The ANN provided a satisfactory MSE during the training; the HW fitting architecture chosen was a 6th-degree polynomial input-output ( Equation 4) to represent the non-linearities since it contributes with the best fit percent in comparison with the other methods.

$$
F(x)=c(1) x^{n}+c(1) x^{n-1}+\ldots+c(n) x+c(n+1)
$$

The Figure 6(a) shows the hysteresis graph with the interpolation of the ANN and HW which are overlapped and for that reason, the Figure $6(\mathrm{~b})$ displays the error in terms of voltage so that the comparison can be appreciated. The ANN behaves significantly better than HW, which has enough distortion. At the peak moment (which occurs at 0.5 seconds), HW has a sudden demeanour that resembles in a sign change; this is expected due to the radical change of slope at the peaks. On the other side, the ANN could manage to fit the hysteresis with only a slight noise during the peak but it can be seen that the error stands on average around the null value. 

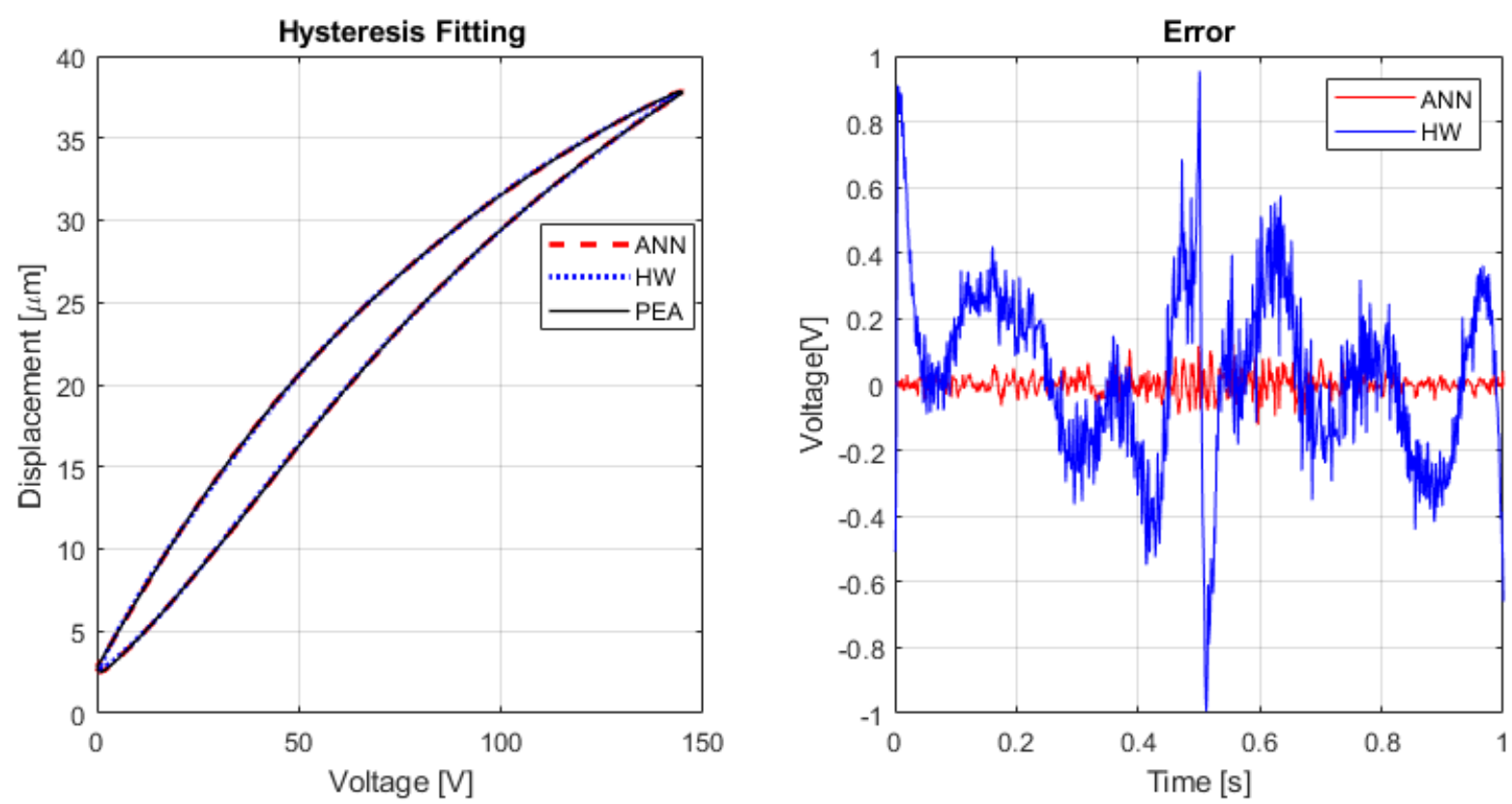

Figure 6. Hysteresis fitting accuracy where (a) corresponds to both models in the fitting and (b) is the error for both models.

\subsection{Guidance control results}

Despite that HW provided a low-grade hysteresis fit, an objective contrast needs to be with a control loop so that the control signal could be a metric as well. The PI parameters were tuned to $K_{p}$ and $K_{i}$ equals to 10 and 100, respectively. The IAE showed values of 0.0384 and 0.0486 which belong to the FF-ANN and FF-HW (both with PI control), respectively. This resembles that the difference is $26 \%$ higher when the ANN was used; however, analysis in depth is presented in the following section.

The Figure 7(a) is the error contrast between the models: the control loop with the FF-HW resulted in less accurate results where the main differences could be seen in special places of the graph. During the first rise, both models managed to behave with a modest difference but at the first peak where the slope shifts, a soar error increment for FF-HW was provoked. During the declining, the framework with FF-HW compensated faster the error although it has more overshoot and with a later undulation. The lower peak at 1 second behaved reciprocally for both models where the one compensated with the ANN had more noise.

Although the control signal from Figure 7(b) looks similar in both cases, the Figure 7(c) and Figure 7(d) displays an amplified view during both peaks. Even if the model FF-HW showed that its less accurate, during the control signal it showed an improvement since the variation is less than the FF-ANN. During the rise, the control signal of the framework with HW had a lower amplitude compared to the contrast and the same happened after the descend. 


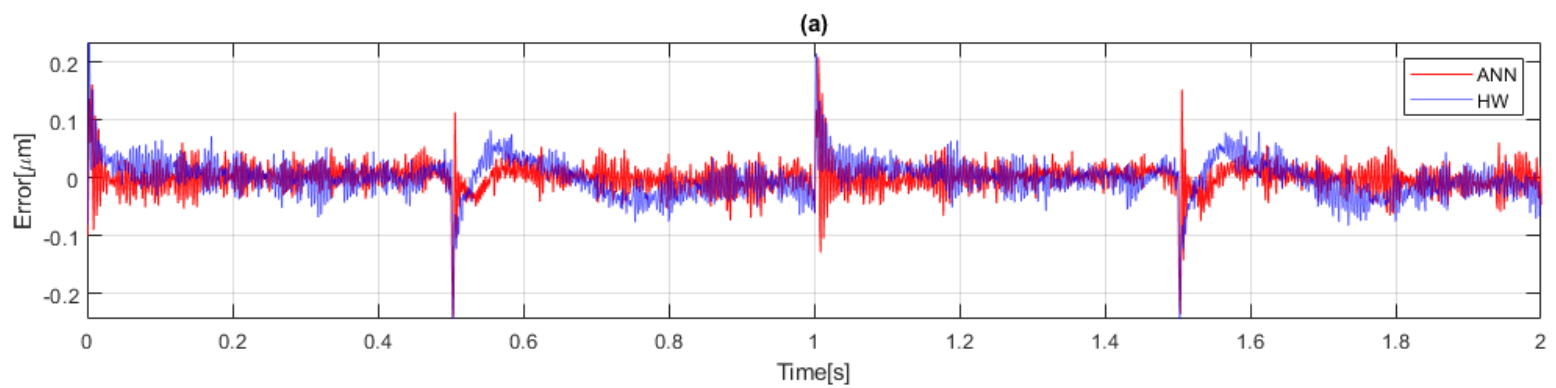

(b)

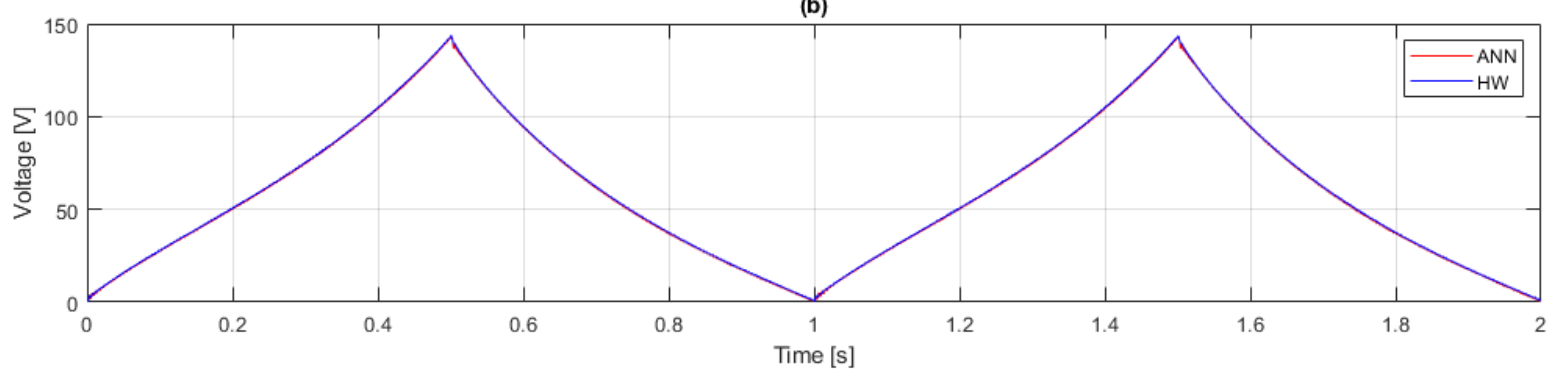

(c)
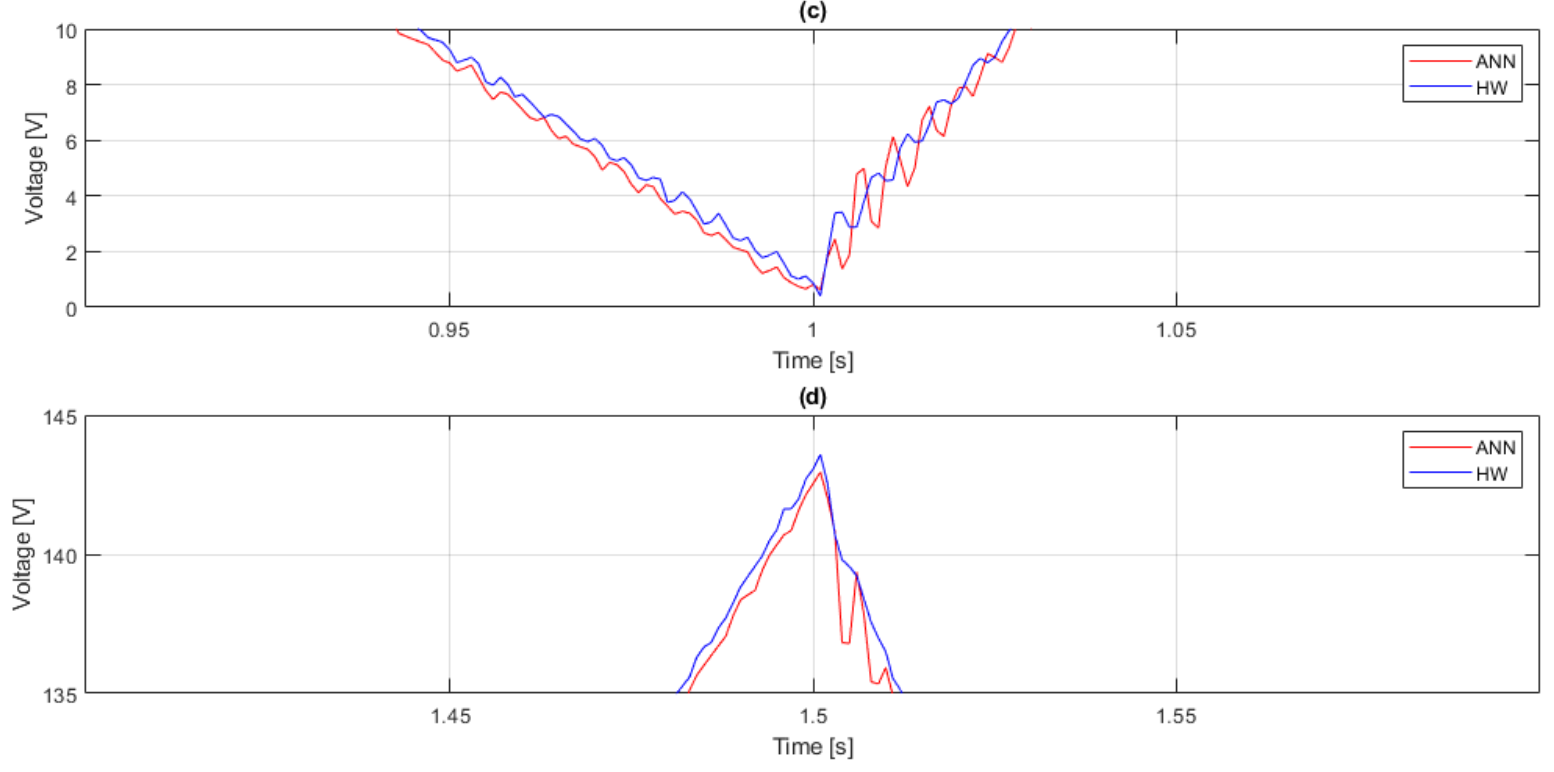

Figure 7. Error comparison and control signal architectures with PI combined with FF-ANN and FF-HW. (a): Error; (b): Control signal; (c): Control signal at the lower peak; (d): Control signal at the top peak.

\section{Conclusions}

During this research, we analysed different mapping alternatives to be used in a feedback FF control architecture. It was found that not only the hysteresis is an undesirable effect and complex to model with conventional tools due to asymmetry (which was present in used PEA) and thus, alternative solutions such as BB can deal with this problem.

The PEA hysteresis was obtained with a triangle wave, which is a complex signal and implies that the control design has to be robust enough. Therefore, a comparison between HW and ANN was done to verify the mapping accuracy. The HW showed low performance in comparison with the ANN since the error had more fluctuation and could carry the peak with certain difficulties. The ANN had an increment in the accuracy which was near the null value of error on average.

However, the control tests showed a different manner since the architecture with the FF-HW had an advantage compared to the FF-ANN. The latter achieved a lower error (which was reflected in the IAE) and performed better than the FF-HW. However, the one with FF-HW showed that the control 
signal was superior: the signal had less undulation and was smoother as well. This implies that the actuation effort is less so that the life-span of the PEA could increase even though the accuracy is lower. Therefore, with this research, the designer has two options to choose which have pros and cons depending on the requirements.

Regarding the options for future research, several combinations can be done. For instance, the PI controller can be replaced with a fractional-proportional-derivative-integral (FPID) so that the complexity is higher. Another option is the usage of a neural PID with advance learning rules. In terms of the FF block, different ANN architectures can be used like long-short-term memory (LSTM); also an optimisation of the HW block can be done by a study using a Hammerstein or a Wiener block so that the calculation is reduced as well as the complexity.

Author Contributions: Conceptualization, O.B. and C.N.; methodology, O.B., I.C. and C.N.; software, C.N.; validation, C.N; formal analysis, O.B. and C.N.; investigation, O.B. and C.N.; resources, C.N.; writing-original draft preparation, C.N., M.D.; writing-review and editing, O.B., C.N., M.D. and M.Y.S.; supervision, O.B. and I.C.; project administration, O.B. and J.V.

Funding: This research was funded by Basque Government and UPV/EHU projects.

Acknowledgments: The authors wish to express their gratitude to the Basque Government through the project SMAR3NAK (ELKARTEK KK-2019/00051), to the Diputación Foral de Álava (DFA) through the project CONAVAUTIN 2 and to the UPV/EHU for supporting this work.

Conflicts of Interest: The authors declare no conflict of interest.

\section{Abbreviations}

The following abbreviations are used in this manuscript:

PEA Piezoelectric Actuator

FF Feed-forward

PI Proportional-Integral

IAE Integral of the absolute error

SMC Sliding Mode Control

BW Bouc-Wen

BB Black-box

ANN Artificial Neural Networks

HW Hammerstein-Wiener

TDNN Time Delay Neural Network

LM Levenberg-Marquardt

MSE Mean Squared Error

\section{References}

1. Gäbel, G.; Millitzer, J.; Atzrodt, H.; Herold, S.; Mohr, A. Development and Implementation of a Multi-Channel Active Control System for the Reduction of Road Induced Vehicle Interior Noise. Actuators 2018, 7, 52.

2. Toledo, J.; Ruiz-Díez, V.; Hernando-García, J.; Sánchez-Rojas, J.L. Piezoelectric Actuators for Tactile and Elasticity Sensing. Actuators 2020, 9, 21.

3. Ai, R.; Monteiro, L.L.S.; Monteiro, P.C.C., Jr.; Pacheco, P.M.C.L.; Savi, M.A. Piezoelectric Vibration-Based Energy Harvesting Enhancement Exploiting Nonsmoothness. Actuators 2019, 8, 25.

4. Hunstig, M. Piezoelectric Inertia Motors-A Critical Review of History, Concepts, Design, Applications, and Perspectives. Actuators 2017, 6, 7.

5. Huang, Y.; Xia, Y.X.; Lin, D.H.; Lim, L.-C. High-Bending-Stiffness Connector (HBSC) and High-Authority Piezoelectric Actuator (HAPA) Made of Such. Actuators 2018, 7, 61.

6. Bertotti, G.; Mayergoyz, I.; Damjanoivc, D. Hysteresis in Piezoelectric and Ferroelectric Materials. In The Science of Hysteresis; Elsevier: Maryland, USA, 2006; Volume 3, pp. 338-448.

7. Frederik, F.; Minorowicz, N. Open loop control of piezoelectric tube transducer. In Mechanical Technology and Materials, 2018, 38, pp. 23-28. 
8. Velasco, J.; Barambones, O.; Calvo, I.; Zubia, J.; Ocariz, I. ; Chouza, A. Sliding Mode Control with Dynamical Correction for Time-Delay Piezoelectric Actuactor Systems. In Materials, 2019, 13, 132.

9. Chouza, A.; Barambones, O.; Calvo, I.; Velasco, J. Sliding Mode-Based Robust Control for Piezoelectric Actuators with Inverse Dynamics Estimation. In Energies, 2019, 12, 943.

10. Song, J.; Der Kiureghian, A. Generalized Bouc-Wen Model for Highly Asymmetric Hysteresis. In Journal of Engineering Mechanics, 2006, 132.

11. Tan, X.; Iyer, R.; Krishnaprasad, P.S. Control of hysteresis: Theory and experimental results. In Proceedings of SPIE, 2001, 4326, pp. 101-112.

12. Peng, J.; Chen, J. A Survey of Modeling and Control of Piezoelectric Actuators. In Modern Mechanical Engineering, 2013, 3, pp. 1-20.

13. Sjöberg, J.; Zhang, Q.; Ljung, L.; Benveniste, A.; Delyon, B.; Glorennec, P.; Hjalmarsson H.; Juditsky, A. Nonlinear black-box modeling in system identification: a unified overview. In Automatica, 1995, 31, pp. 1691-1724.

14. Napole, C.; Barambones, O.; Calvo, I.; Velasco, J. Feedforward Compensation Analysis of Piezoelectric Actuators Using Artificial Neural Networks with Conventional PID Controller and Single-Neuron PID Based on Hebb Learning Rules. In Energies, 2020, 13, 3929.

15. Wills, A.; Schön, T.; Ljung, L.; Ninness, B. Identification of Hammerstein-Wiener Models. In Automatica, 2012, 49, pp. 70-81.

16. Wang, Y.; Ho, J.; Jiang Y. A self-positioning linear actuator based on a piezoelectric slab with multiple pads. In Mechanical Systems and Signal Processing, 2021, 150, 107245.

17. Qin, Y.; Duan, H. Single-Neuron Adaptive Hysteresis Compensation of Piezoelectric Actuator Based on Hebb Learning Rules. In Micromachines, 2020, 11, pp. 84.

18. Waibel, A.; Hanazana, T.; Hinton, G.; Shikano, K.; Lang, K.J. Phoneme recognition using time delay neural networks. In IEEE Trans. Acoust. Speech Signal Process, 1989, 37, 328-339.

(C) 2020by the authors. Licensee MDPI, Basel, Switzerland. This article is an open access article distributed under the terms and conditions of the Creative Commons Attribution (CC BY) license (http://creativecommons.org/licenses/by/4.0/). 